\title{
"ADDITION" THEOREMS FOR SOME $q$-EXPONENTIAL AND $q$-TRIGONOMETRIC FUNCTIONS
}

\section{S. K. Suslov}

ABSTRACT. We derive $q$-analogs of addition theorems for some $q$-exponential and $q$-trigonometric functions.

\section{Introduction}

Exponential and trigonometric functions can be introduced on the basis of the simple second-order differential equation

$$
\sigma u^{\prime \prime}+\lambda u=0
$$

where $\sigma$ and $\lambda$ are constants. If $\lambda / \sigma=-\alpha^{2}$, solutions of (1.1) are

$$
\begin{aligned}
u_{1} & =e^{\alpha x}=\sum_{k=0}^{\infty} \frac{(\alpha x)^{k}}{k !}, & u_{2} & =e^{-\alpha x}, \\
u_{3} & =e^{\alpha(x+y)}, & u_{4} & =e^{-\alpha(x+y)}, \\
u_{5} & =\cosh \alpha x=\frac{1}{2}\left(e^{\alpha x}+e^{-\alpha x}\right), & u_{6} & =\sinh \alpha x=\frac{1}{2}\left(e^{\alpha x}-e^{-\alpha x}\right), \\
u_{7} & =\cosh \alpha(x+y), & u_{8} & =\sinh \alpha(x+y) .
\end{aligned}
$$

When $\lambda / \sigma=\omega^{2}$, solutions of (1.1) are related to trigonometric functions

$$
\begin{array}{ll}
u_{1}=e^{i \omega x}=\cos \omega x+i \sin \omega x, & u_{2}=e^{-i \omega x}=\cos \omega x-i \sin \omega x, \\
u_{3}=e^{i \omega(x+y)}, & u_{4}=e^{-i \omega(x+y)}, \\
u_{5}=\cos \omega x, & u_{6}=\sin \omega x, \\
u_{7}=\cos \omega(x+y), & u_{8}=\sin \omega(x+y) .
\end{array}
$$

A second-order differential equation may have only two linearly independent solutions. This leads in a natural way to classical addition theorems for the exponential and trigonometric functions. For example, from (1.2) and (1.3),

$$
e^{\alpha(x+y)}=A e^{\alpha x}+B e^{-\alpha x}
$$

where $A$ and $B$ are some constants independent of $x$. Differentiating (1.10) with respect to $x$,

$$
e^{\alpha(x+y)}=A e^{\alpha x}-B e^{-\alpha x}
$$

Received June 18, 1996, revised August 27, 1996.

1991 Mathematics Subject Classification: Primary 33D15; Secondary 33B10.

Key words and phrases: exponential and trigonometric functions, addition theorems, $q$-exponential and $q$-trigonometric functions, basic hypergeometric series. 
and, therefore,

$$
e^{\alpha(x+y)}=A e^{\alpha x} .
$$

Letting $x=0$, one gets $A=e^{\alpha y}$ and, finally, arrives at the addition theorem for the exponential function

$$
e^{\alpha(x+y)}=e^{\alpha x} e^{\alpha y} .
$$

One can prove (1.13) by direct series manipulations with the help of the binomial theorem. Another well-known fact is that the exponential function $e^{\alpha x}$ is the only measurable solution of the functional equation

$$
f(x+y)=f(x) f(y) .
$$

Similar arguments lead to addition theorems for trigonometric functions

$$
\begin{aligned}
& \cos \omega(x+y)=\cos \omega x \cos \omega y-\sin \omega x \sin \omega y, \\
& \sin \omega(x+y)=\sin \omega x \cos \omega y+\cos \omega x \sin \omega y .
\end{aligned}
$$

There is a great deal of interest these days in $q$-analogs of important classical formulas. A few $q$-analogs of exponential and trigonometric functions are known. F. $\mathrm{H}$. Jackson's $q$-analogs of the exponential function were studied in detail (see [9]) and have been found useful in many applications (see, for example, [8, 12] and references therein). Some new $q$-exponential and $q$-trigonometric functions were introduced recently $[10,15]$ (cf. also [6], and see [15] for a nice review). In the present paper we discuss these new $q$-exponential and $q$-trigonometric functions on the basis of a difference analog of (1.1). We derive $q$-versions of the addition theorems (1.13) and (1.15) $-(1.16)$ for these functions. It is worth mentioning that more $q$-analogs of exponential functions and some of their extensions have appeared as kernels of $q$-Fourier transformations in $[2,3,4,16]$.

The paper is organized as follows. In Section 2 we discuss a general $q$-difference analog of the differential equation (1.1) and find its solutions. The analog of the exponential function on $q$-quadratic lattice is introduced in Section 3 where we give the first proof of an "addition" theorem for that function. The corresponding $q$ trigonometric functions are studied in Section 4. An important special case of $q$ linear grids is discussed independently in Sections 5 and 6. In Section 7 we derive a general product formula for $q$-exponential functions on $q$-quadratic grids. This gives an alternative proof of the "addition" theorems. The special case of the linear lattice is discussed in Section 8. Here the addition theorem has the usual form (1.13), and we transform the "difference" exponential function to the classical one. We close the paper by considering an application of the "addition" theorem in Section 9.

\section{The difference equation and its solutions}

The difference analog of (1.1) on nonuniform lattices is

$$
\sigma \frac{\Delta}{\nabla x_{1}(z)}\left(\frac{\nabla u(z)}{\nabla x(z)}\right)+\lambda u(z)=0
$$

where

$$
x(z)=C_{1} q^{-z}+C_{2} q^{z}, \quad x_{1}(z)=x\left(z+\frac{1}{2}\right)
$$


$\sigma, \lambda, C_{1}, C_{2}$, and $q$ are constants, and $\Delta f(z)=\nabla f(z+1)=f(z+1)-f(z)$. Equation (2.1) is the simplest case of the general difference equation of hypergeometric type on nonuniform lattices (see $[14,18]$ for details).

Solutions of difference equations of hypergeometric type can be found by the extended power series method [6]. The most general formal "power series" solution of (2.1) has the form

$$
u(z, s)=\sum_{n=0}^{\infty} \frac{(-\lambda / \sigma)^{n / 2}}{\gamma(n) !}\left[x(z)-x\left(s+\frac{n-1}{2}\right)\right]^{(n)}
$$

where $s$ is an additional "free" parameter,

$$
\gamma(n) !:=\gamma(1) \gamma(2) \cdots \gamma(n), \quad \gamma(k):=\frac{q^{k / 2}-q^{-k / 2}}{q^{1 / 2}-q^{-1 / 2}}
$$

and the "generalized power" is defined as

$$
[x(z)-x(v)]^{(n)}:=\prod_{k=0}^{n-1}[x(z)-x(v-k)] .
$$

Using the difference-differentiation formula [18]

$$
\frac{\delta}{\delta x(z)}[x(z)-x(v)]^{(n)}=\gamma(n)[x(z)-x(v-1 / 2)]^{(n-1)}
$$

where $\delta f(x)=f(z+1 / 2)-f(z-1 / 2)$, one can show that the series (2.3) satisfies the equation

$$
\frac{\delta u(z, s)}{\delta x(z)}=\left(-\frac{\lambda}{\sigma}\right)^{1 / 2} u(z, s) .
$$

Applying the operator $\delta / \delta x(z)$ one more time, one gets (2.1).

The "even" and "odd" parts of (2.3),

$$
\begin{aligned}
& u_{+}(z, s)=\sum_{k=0}^{\infty} \frac{(-\lambda / \sigma)^{k}}{\gamma(2 k) !}[x(z)-x(s+k-1 / 2)]^{(2 k)}, \\
& u_{-}(z, s)=\sum_{k=0}^{\infty} \frac{(-\lambda / \sigma)^{k+1 / 2}}{\gamma(2 k+1) !}[x(z)-x(s+k)]^{(2 k+1)}
\end{aligned}
$$

both satisfy equation (2.1) as well. Also,

$$
\frac{\delta u_{+}(z, s)}{\delta x(z)}=\left(-\frac{\lambda}{\sigma}\right)^{1 / 2} u_{-}(z, s), \quad \frac{\delta u_{-}(z, s)}{\delta x(z)}=\left(-\frac{\lambda}{\sigma}\right)^{1 / 2} u_{+}(z, s) .
$$

Solutions (2.3), (2.8)-(2.9) can be represented in terms of basic hypergeometric series. Indeed,

$$
\left[x(z)-x\left(s+\frac{n-1}{2}\right)\right]^{(n)}=\left(-C_{1}\right)^{n} q^{-n s}\left(q^{s-z-(n-1) / 2}, \mu q^{s+z-(n-1) / 2} ; q\right)_{n},
$$


or

$$
\begin{aligned}
{[x(z)} & -x(s+k-1 / 2)]^{(2 k)} \\
& =\left(C_{1} C_{2}\right)^{k} q^{-k^{2}}\left(q^{1 / 2+z-s}, q^{1 / 2-z+s}, \mu q^{1 / 2+z+s}, q^{1 / 2-z-s} / \mu ; q\right)_{k},
\end{aligned}
$$

and

$$
\begin{aligned}
& {[x(z)-x(s+k)]^{(2 k+1)}} \\
& \quad=[x(z)-x(s)]\left(C_{1} C_{2}\right)^{k} q^{-k(k+1)}\left(q^{1+z-s}, q^{1-z+s}, \mu q^{1+z+s}, q^{1-z-s} / \mu ; q\right)_{k}
\end{aligned}
$$

where $\mu=C_{2} / C_{1}$.

We follow the notation for $q$-shifted factorials of Gasper and Rahman [9]

$$
\begin{aligned}
& (a ; q)_{0}:=1, \quad(a ; q)_{n}:=\prod_{k=0}^{n-1}\left(1-a q^{k}\right), \\
& \left(a_{1}, a_{2}, \ldots, a_{m} ; q\right)_{n}:=\prod_{l=1}^{m}\left(a_{l} ; q\right)_{n} .
\end{aligned}
$$

Here $n=1,2, \ldots$, or $\infty$, when $|q|<1$. The basic hypergeometric series is defined by

$$
\begin{aligned}
{ }_{r} \varphi_{s}(\zeta) & :={ }_{r} \varphi_{s}\left(\begin{array}{c}
a_{1}, a_{2}, \ldots, a_{r} \\
b_{1}, \ldots, b_{s}
\end{array} ; q, \zeta\right) \\
& =\sum_{n=0}^{\infty} \frac{\left(a_{1}, a_{2}, \ldots, a_{r} ; q\right)_{n}}{\left(q, b_{1}, \ldots, b_{s} ; q\right)_{n}}\left((-1)^{n} q^{n(n-1) / 2}\right)^{1+s-r} \zeta^{n} .
\end{aligned}
$$

See [9] for details.

Solution (2.3) is the sum of two ${ }_{4} \varphi_{3}$ 's,

$$
\begin{aligned}
u(z, s)={ }_{4} \varphi_{3} & \left(\begin{array}{c}
q^{1 / 2+z-s}, q^{1 / 2-z+s}, \mu q^{1 / 2+z+s}, q^{1 / 2-z-s} / \mu \\
-q, q^{1 / 2},-q^{1 / 2}
\end{array} ;,-\frac{\lambda C_{1} C_{2}(1-q)^{2}}{\sigma q^{1 / 2}}\right) \\
+ & \left(-\frac{\lambda}{\sigma}\right)^{1 / 2}[x(z)-x(s)] \\
& \times{ }_{4} \varphi_{3}\left(\begin{array}{c}
q^{1+z-s}, q^{1-z+s}, \mu q^{1+z+s}, q^{1-z-s} / \mu \\
-q, q^{3 / 2},-q^{3 / 2}
\end{array} ;,-\frac{\lambda C_{1} C_{2}(1-q)^{2}}{\sigma q^{1 / 2}}\right) .
\end{aligned}
$$

Both ${ }_{4} \varphi_{3}$ 's converge absolutely when $\left|C_{1} C_{2}(1-q)^{2} q^{-1 / 2} \lambda / \sigma\right|<1$. Analytic continuation of (2.17) will be discussed in Section 7.

\section{Analogs of exponential functions on q-quadratic grids}

Function (2.17) may be considered as a general $q$-analog of the exponential function on $q$-linear and $q$-quadratic grids. Let us specify parameters in a convenient way and 
introduce

$$
\begin{aligned}
\mathcal{E}(x, y):= & \mathcal{E}_{q}(x, y ; \alpha) \\
= & \frac{\left(\alpha^{2} ; q^{2}\right)_{\infty}}{\left(q \alpha^{2} ; q^{2}\right)_{\infty}} \sum_{n=0}^{\infty} \frac{q^{n^{2} / 4}}{(q ; q)_{n}} e^{-i n \varphi}\left(-q^{(1-n) / 2} e^{i \varphi+i \theta},-q^{(1-n) / 2} e^{i \varphi-i \theta} ; q\right)_{n} \alpha^{n} \\
= & \left.\frac{\left(\alpha^{2} ; q^{2}\right)_{\infty}}{\left(q \alpha^{2} ; q^{2}\right)_{\infty}} \quad ; q, \alpha^{2}\right) \\
& \times\left[{ }_{4} \varphi_{3}\left(-q^{1 / 2} e^{i \theta+i \varphi},-q^{1 / 2} e^{i \theta-i \varphi},-q^{1 / 2} e^{i \varphi-i \theta},-q^{1 / 2} e^{-i \theta-i \varphi}-q, q^{1 / 2},-q^{1 / 2} \quad ; q, \alpha^{2}\right)\right] \\
& +\left(\frac{2 q^{1 / 4}}{1-q}\right) \alpha(\cos \theta+\cos \varphi) \\
& \times{ }_{4} \varphi_{3}\left(-q e^{i \theta+i \varphi},-q e^{i \theta-i \varphi},-q e^{i \varphi-i \theta},-q e^{-i \theta-i \varphi} \quad-q, q^{3 / 2},-q^{3 / 2} \quad\right.
\end{aligned}
$$

as the $q$-version of $e^{\alpha(x+y)}$ on a $q$-quadratic grid. Here $x=\frac{1}{2}\left(q^{z}+q^{-z}\right)=\cos \theta$, $q^{z}=e^{i \theta}$, and $y=\frac{1}{2}\left(q^{s}+q^{-s}\right)=\cos \varphi, q^{s}=e^{i \varphi}$. We assume that $|\alpha|<1$. One can see that

$$
\lim _{q \rightarrow 1^{-}} \mathcal{E}_{q}\left(x, y ; \frac{1}{2} \alpha(1-q)\right)=e^{\alpha(x+y)} .
$$

With a different normalization, these functions were introduced originally by Ismail and Zhang [10],

$$
\begin{aligned}
\mathcal{E}_{q}(x ; a, b):= & \sum_{n=0}^{\infty} \frac{q^{n^{2} / 4}}{(q ; q)_{n}}\left(a q^{(1-n) / 2} e^{i \theta}, a q^{(1-n) / 2} e^{-i \theta} ; q\right)_{n} b^{n} \\
= & { }_{4} \varphi_{3}\left(\begin{array}{c}
\left.a^{-1} q^{1 / 2} e^{i \theta}, a^{-1} q^{1 / 2} e^{-i \theta}, a q^{1 / 2} e^{i \theta}, a q^{1 / 2} e^{-i \theta} ; q, a^{2} b^{2}\right) \\
-q, q^{1 / 2},-q^{1 / 2}
\end{array}\right) \\
+ & \left(\frac{q^{1 / 4}}{1-q}\right) b\left(1-2 a \cos \theta+a^{2}\right) \\
& \times{ }_{4} \varphi_{3}\left(\begin{array}{c}
a^{-1} q e^{i \theta}, a^{-1} q e^{-i \theta}, a q e^{i \theta}, a q e^{-i \theta} \\
-q, q^{3 / 2},-q^{3 / 2}
\end{array} ; q, a^{2} b^{2}\right) .
\end{aligned}
$$

Their definition corresponds to the following choice of parameters in (2.17): $C_{1}=$ $C_{2}=1 / 2, \mu=1, q^{s}=a, q^{z}=e^{i \theta}$, and $\lambda / \sigma=-4 q^{1 / 2}(1-q)^{-2} a^{2} b^{2}$. Comparing (3.3) with (3.1), one gets

$$
\mathcal{E}_{q}(\cos \theta, \cos \varphi ; \alpha)=\frac{\left(\alpha^{2} ; q^{2}\right)_{\infty}}{\left(q \alpha^{2} ; q^{2}\right)_{\infty}} \mathcal{E}_{q}\left(\cos \theta ;-e^{i \varphi}, \alpha e^{-i \varphi}\right)
$$

Ismail and Zhang also proved that

$$
\lim _{q \rightarrow 1^{-}} \mathcal{E}_{q}(x ; a, b(1-q))=\exp \left(\left(1+a^{2}-2 a x\right) b\right)
$$

(cf. (3.2)). Ismail and Zhang expanded the $\mathcal{E}_{q}$ in terms of " $q$-spherical harmonics" [10], and later, together with Rahman [11], they extended this $q$-analog of the expansion 
formula of the plane wave from $q$-ultraspherical polynomials to continuous $q$-Jacobi polynomials.

Another choice of the parameters in (2.17) was discussed by Rahman [15].

Let us also consider the special case of (3.1) (or (3.3)),

$$
\begin{aligned}
\mathcal{E}(x):= & \mathcal{E}_{q}(x ; \alpha)=\mathcal{E}_{q}(x, 0 ; \alpha) \\
= & \frac{\left(\alpha^{2} ; q^{2}\right)_{\infty}}{\left(q \alpha^{2} ; q^{2}\right)_{\infty}} \sum_{n=0}^{\infty} \frac{q^{n^{2} / 4}}{(q ; q)_{n}}(-i)^{n}\left(-i q^{(1-n) / 2} e^{i \theta},-i q^{(1-n) / 2} e^{-i \theta} ; q\right)_{n} \alpha^{n} \\
= & \frac{\left(\alpha^{2} ; q^{2}\right)_{\infty}}{\left(q \alpha^{2} ; q^{2}\right)_{\infty}}\left[{ }_{2} \varphi_{1}\left(\begin{array}{c}
-q e^{2 i \theta},-q e^{-2 i \theta} ; q^{2}, \alpha^{2} \\
q \quad
\end{array}\right)\right. \\
& \left.\quad+\left(\frac{2 q^{1 / 4}}{1-q}\right) \alpha \cos \theta_{2} \varphi_{1}\left(\begin{array}{c}
-q^{2} e^{2 i \theta},-q^{2} e^{-2 i \theta} ; q^{2}, \alpha^{2} \\
q^{3}
\end{array}\right)\right],
\end{aligned}
$$

as the $q$-analog of $e^{\alpha x}$.

The following properties hold:

$$
\begin{aligned}
& \mathcal{E}_{q}(0,0 ; \alpha)=\mathcal{E}_{q}(0 ; \alpha)=1, \\
& \mathcal{E}_{q}(x, y ; \alpha)=\mathcal{E}_{q}(y, x ; \alpha) .
\end{aligned}
$$

The functions $\mathcal{E}(x, y)$ and $\mathcal{E}(x)$ both satisfy the equation

$$
\frac{\delta u}{\delta x}=\frac{2 q^{1 / 4}}{1-q} \alpha u
$$

which is the $q$-version of

$$
\frac{d}{d x} e^{\alpha(x+y)}=\alpha e^{\alpha(x+y)} .
$$

Our next aim is to prove the following version of the addition theorem for the $q$-exponential functions (3.1) and (3.5).

\section{Theorem 3.1.}

$$
\mathcal{E}(x, y)=\mathcal{E}(x) \mathcal{E}(y) .
$$

Proof. Functions $\mathcal{E}(x, y), \mathcal{E}(x)$, and $\mathcal{E}(-x)$ satisfy the second-order difference equation

$$
\frac{\delta^{2} u}{\delta x^{2}}=\left(\frac{2 q^{1 / 4}}{1-q} \alpha\right)^{2} u
$$

Therefore,

$$
\mathcal{E}(x, y)=A \mathcal{E}(x)+B \mathcal{E}(-x)
$$

where $A=A(z, s)$ and $B=B(z, s)$ are, generally speaking, some functions of period 1 in $z$. Applying $\delta / \delta x(z)$ to both sides of (3.11),

$$
\mathcal{E}(x, y)=A \mathcal{E}(x)-B \mathcal{E}(-x)
$$

or

$$
\mathcal{E}(x, y)=A \mathcal{E}(x)
$$


By their definitions, functions $\mathcal{E}(x, y)$ and $\mathcal{E}(x)$ have the natural period $T=2 \pi i / \log q$ in $z$, so

$$
A(z, s)=\frac{\mathcal{E}(x, y)}{\mathcal{E}(x)}
$$

is a doubly periodic analytic function in $z$.

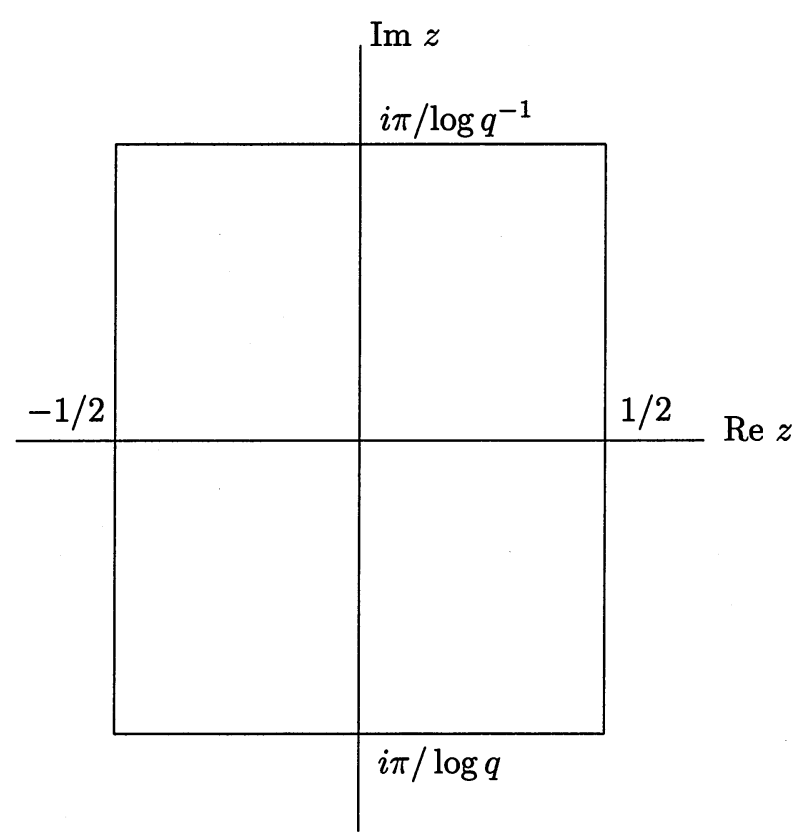

FIGURE 1

When $\alpha \rightarrow 0, \mathcal{E}(x)=1+O(\alpha)$, and, therefore, for sufficiently small $|\alpha|$, the function $\mathcal{E}(x)$ does not have zeros in the parallelogram in Figure 1. Thus, $A(z, s)$ is an entire doubly periodic function that is a constant by Liouville's theorem. One can find this constant by choosing $x=0$ (or $\theta=\pi / 2$ ) in (3.13). This results in (3.9).

In Section 7, we shall give another proof of (3.9).

In the proof of Theorem 3.1, we have en route established the following fact.

Corollary 3.2. The function $u=\mathcal{E}(x, y)$ is the only analytic solution of the difference equation (3.8) on the q-quadratic lattice $x=\frac{1}{2}\left(q^{z}+q^{-z}\right)$ which satisfies the initial condition $u(0)=\mathcal{E}(y)$ and does not have poles in the parallelogram in Figure 1.

Proof. Suppose that $u=u(x, y)$ is some solution of (3.8) satisfying the above hypotheses. Then, in the same manner,

$$
u(x, y)=A \mathcal{E}(x)
$$

where $A$ is a constant. Letting $x=0$, we obtain

$$
u(x, y)=\mathcal{E}(x) \mathcal{E}(y)=\mathcal{E}(x, y)
$$

by (3.9). 
As we already have mentioned in the Introduction, the function $\exp (\alpha x)$ is the only measurable solution of (1.14). Solutions of the functional equation (3.9) are, obviously, not unique. But the following result may be considered as a characterization of the $q$-exponential function (3.1).

Theorem 3.3. The function $\mathcal{E}(x, y)$ is the only analytic solution of the partial difference equation

$$
\frac{\delta F(x, y)}{\delta x}=\frac{\delta F(x, y)}{\delta y}
$$

on the q-quadratic lattices $x=\frac{1}{2}\left(q^{z}+q^{-z}\right)$ and $y=\frac{1}{2}\left(q^{s}+q^{-s}\right)$ of the form

$$
F(x, y)=f(x) f(y), \quad f(0)=1,
$$

provided that the function $f(x)$ does not have poles in the parallelogram in Figure 1.

Proof. Substituting (3.18) into (3.17) gives (3.8) for the function $f(x)$ where $\alpha$ is a constant of separation of variables. But the only solution of this equation satisfying $f(0)=1$ under the conditions of the theorem is $\mathcal{E}(x)$ (cf. Corollary 3.2 when $y=0$ ). This gives $F(x, y)=\mathcal{E}(x, y)$ by $(3.18)$ and (3.9).

\section{Analogs of trigonometric functions on $q$-quadratic grids}

Let $\alpha=i \omega$ in (3.1). Then

$$
\mathcal{E}_{q}(x, y ; i \omega)=C(x, y)+i S(x, y)
$$

where

$$
\begin{aligned}
& C(x, y):=C_{q}(x, y ; \omega) \\
& =\frac{\left(-\omega^{2} ; q^{2}\right)_{\infty}}{\left(-q \omega^{2} ; q^{2}\right)_{\infty}}{ }_{4} \varphi_{3}\left(\begin{array}{c}
-q^{1 / 2} e^{i \theta+i \varphi},-q^{1 / 2} e^{i \theta-i \varphi},-q^{1 / 2} e^{i \varphi-i \theta},-q^{1 / 2} e^{-i \theta-i \varphi} \\
-q, q^{1 / 2},-q^{1 / 2}
\end{array} ;,-\omega^{2}\right)
\end{aligned}
$$

and

$$
\begin{aligned}
S(x, y):= & S_{q}(x, y ; \omega) \\
= & \frac{\left(-\omega^{2} ; q^{2}\right)_{\infty}}{\left(-q \omega^{2} ; q^{2}\right)_{\infty}} \frac{2 q^{1 / 4}}{1-q} \omega(\cos \theta+\cos \varphi) \\
& \quad \times{ }_{4} \varphi_{3}\left(\begin{array}{c}
-q e^{i \theta+i \varphi},-q e^{i \theta-i \varphi},-q e^{i \varphi-i \theta},-q e^{-i \theta-i \varphi} \\
-q, q^{3 / 2},-q^{3 / 2}
\end{array} ;,-\omega^{2}\right)
\end{aligned}
$$

are the $q$-versions of $\cos \omega(x+y)$ and $\sin \omega(x+y)$, respectively. Indeed,

$$
\begin{aligned}
& \lim _{q \rightarrow 1^{-}} C_{q}\left(x, y ; \frac{1}{2} \omega(1-q)\right)=\cos \omega(x+y), \\
& \lim _{q \rightarrow 1^{-}} S_{q}\left(x, y ; \frac{1}{2} \omega(1-q)\right)=\sin \omega(x+y) .
\end{aligned}
$$

In the special case $y=0(\varphi=\pi / 2)$ of (4.1)-(4.3), we get

$$
\mathcal{E}_{q}(x ; i \omega)=C(x)+i S(x)
$$


where

$$
\begin{aligned}
C(x): & =C_{q}(x ; \omega)=C_{q}(x, 0 ; \omega) \\
& =\frac{\left(-\omega^{2} ; q^{2}\right)_{\infty}}{\left(-q \omega^{2} ; q^{2}\right)_{\infty}}{ }_{2} \varphi_{1}\left(\begin{array}{c}
-q e^{2 i \theta},-q e^{-2 i \theta} \\
q
\end{array} q^{2},-\omega^{2}\right)
\end{aligned}
$$

and

$$
\begin{aligned}
S(x) & :=S_{q}(x ; \omega)=S_{q}(x, 0 ; \omega) \\
& =\frac{\left(-\omega^{2} ; q^{2}\right)_{\infty}}{\left(-q \omega^{2} ; q^{2}\right)_{\infty}} \frac{2 q^{1 / 4}}{1-q} \omega \cos \theta_{2} \varphi_{1}\left(\begin{array}{c}
-q^{2} e^{2 i \theta},-q^{2} e^{-2 i \theta} \\
q^{3}
\end{array} q^{2},-\omega^{2}\right)
\end{aligned}
$$

are analogs of $\cos \omega x$ and $\sin \omega x$, respectively $[6,10]$.

The $q$-trigonometric functions (4.2)-(4.3) and (4.5)-(4.6) satisfy the differencedifferentiation formulas [10]

$$
\frac{\delta}{\delta x} C(x, y)=-\frac{2 q^{1 / 4}}{1-q} \omega S(x, y)
$$

and

$$
\frac{\delta}{\delta x} S(x, y)=\frac{2 q^{1 / 4}}{1-q} \omega C(x, y)
$$

which are the $q$-analogs of

$$
\frac{d}{d x} \cos \omega(x+y)=-\omega \sin \omega(x+y), \quad \frac{d}{d x} \sin \omega(x+y)=\omega \cos \omega(x+y) .
$$

The following $q$-versions of addition theorems (1.15)-(1.16) are valid.

\section{Theorem 4.1.}

$$
\begin{gathered}
C(x, y)=C(x) C(y)-S(x) S(y), \\
S(x, y)=S(x) C(y)+C(x) S(y) .
\end{gathered}
$$

Proof. The analog of $\exp (i \omega(x+y))=\exp (i \omega x) \exp (i \omega y)$ is

$$
\mathcal{E}_{q}(x, y ; i \omega)=\mathcal{E}_{q}(x ; i \omega) \mathcal{E}_{q}(y ; i \omega) .
$$

Substituting (4.1) and (4.4) in (4.11) one gets (4.9)-(4.10).

Some special cases of (4.9)-(4.10) are

$$
\begin{aligned}
C(x, x) & =C^{2}(x)-S^{2}(x), \\
S(x, x) & =2 S(x) C(x), \\
C(x,-x) & =C^{2}(x)+S^{2}(x),
\end{aligned}
$$

and

$$
\begin{aligned}
& C(x,-x)+C(x, x)=2 C^{2}(x) \\
& C(x,-x)-C(x, x)=2 S^{2}(x)
\end{aligned}
$$


which are the analogs of the well-known trigonometric identities

$$
\begin{aligned}
& \cos 2 \omega x=\cos \omega x-\sin \omega x \\
& \sin 2 \omega x=2 \sin \omega x \cos \omega x, \\
& \cos ^{2} \omega x+\sin ^{2} \omega x=1
\end{aligned}
$$

and

$$
1+\cos 2 \omega x=2 \cos ^{2} \omega x, \quad 1-\cos 2 \omega x=2 \sin ^{2} \omega x,
$$

respectively. One can see that $C(x,-x)$ is not a constant, which is what we get in the classical case, but is a nonnegative function due to (4.14) if $C(x)$ and $S(x)$ are real.

\section{Some $q$-exponential and $q$-trigonometric functions on $q$-linear grids}

Taking the limit $C_{2} \rightarrow 0$ with $C_{1}=1$ and $\alpha^{2}=q^{-1 / 2}(1-q)^{2} \lambda / \sigma$ in (2.17), one can introduce

$$
\begin{aligned}
\varepsilon(x, y):= & \varepsilon_{q}(x, y ; \alpha) \\
={ }_{3} \varphi_{3}\left(\begin{array}{c}
-q^{1 / 2} x / y,-q^{1 / 2} y / x, 0 \\
-q, q^{1 / 2},-q^{1 / 2} ; q,-\alpha^{2} x y
\end{array}\right) & \quad+\frac{\alpha}{1-q}(x+y)_{3} \varphi_{3}\left(\begin{array}{c}
-q x / y,-q y / x, 0 \\
-q, q^{3 / 2},-q^{3 / 2} ; q,-\alpha^{2} q^{1 / 2} x y
\end{array}\right) \\
= & \sum_{n=0}^{\infty} \frac{q^{n(n-1) / 4}}{(q ; q)_{n}}\left(-q^{(1-n) / 2} y / x ; q\right)_{n}(\alpha x)^{n}
\end{aligned}
$$

as the $q$-version of $e^{\alpha(x+y)}$ on the $q$-linear grid. This function has recently appeared in [15]. Also,

$$
\begin{aligned}
\varepsilon(x) & :=\varepsilon_{q}(x ; \alpha)=\varepsilon_{q}(x, 0 ; \alpha) \\
& ={ }_{1} \varphi_{1}\left(\begin{array}{l}
0 \\
q
\end{array} ; q^{2},-\alpha^{2} q^{1 / 2} x^{2}\right)+\frac{\alpha}{1-q} x_{1} \varphi_{1}\left(\begin{array}{l}
0 \\
q
\end{array} ; q^{2},-\alpha^{2} q^{3 / 2} x^{2}\right) \\
& =\sum_{n=0}^{\infty} \frac{q^{n(n-1) / 4}}{(q ; q)_{n}}(\alpha x)^{n}
\end{aligned}
$$

is a $q$-version of $e^{\alpha x}$, see $[6,7,15]$.

In the next section, we shall give a direct proof of the following "addition" theorem found by Rahman [15] in the case of the $q$-linear lattice.

\section{Theorem 5.1.}

$$
\varepsilon(x, y)=\varepsilon(x) \varepsilon(y) .
$$

This theorem also can be proved on the basis of the difference equation in the same manner as Theorem 3.1. Analogs of Corollary 3.2 and Theorem 3.3 are valid.

Letting $\alpha=i \omega$ in (5.1)-(5.2), one gets

$$
\varepsilon_{q}(x, y ; i \omega)=c(x, y)+i s(x, y)
$$


where

$$
\begin{aligned}
& c(x, y):=c_{q}(x, y ; \omega) \\
& ={ }_{3} \varphi_{3}\left(\begin{array}{c}
-q^{1 / 2} x / y,-q^{1 / 2} y / x, 0 \\
-q, q^{1 / 2},-q^{1 / 2}
\end{array} ; q, \omega^{2} x y\right)
\end{aligned}
$$

and

$$
\begin{aligned}
s(x, y): & =s_{q}(x, y ; \omega) \\
& =\frac{\omega}{1-q}(x+y)_{3} \varphi_{3}\left(\begin{array}{c}
-q x / y,-q y / x, 0 \\
-q, q^{3 / 2},-q^{3 / 2} ; q, \omega^{2} q^{1 / 2} x y
\end{array}\right)
\end{aligned}
$$

as $q$-versions of $\cos \omega(x+y)$ and $\sin \omega(x+y)$ on $q$-linear grids, respectively.

In the same manner,

$$
\varepsilon_{q}(x ; i \omega)=c(x)+i s(x)
$$

where

$$
\begin{aligned}
c(x) & :=c_{q}(x ; \omega)=c_{q}(x, 0 ; \omega) \\
& ={ }_{1} \varphi_{1}\left(\begin{array}{l}
0 \\
q^{\prime}
\end{array} q^{2}, \omega^{2} q^{1 / 2} x^{2}\right)
\end{aligned}
$$

and

$$
\begin{aligned}
s(x) & :=s_{q}(x ; \omega)=s_{q}(x, 0 ; \omega) \\
& =\frac{\omega}{1-q} x_{1} \varphi_{1}\left(\begin{array}{c}
0 \\
q^{3}
\end{array} ; q^{2}, \omega^{2} q^{3 / 2} x^{2}\right)
\end{aligned}
$$

are the $q$-analogs of $\cos \omega x$ and $\sin \omega x$ [13].

As a consequence of Theorem 5.1, we have the following "addition" formulas for $q$-trigonometric functions (5.5)-(5.6) and (5.8)-(5.9).

\section{Theorem 5.2.}

$$
\begin{aligned}
& c(x, y)=c(x) c(y)-s(x) s(y), \\
& s(x, y)=s(x) c(y)+c(x) s(y) .
\end{aligned}
$$

Special cases of these relations analogous to (4.12)-(4.16) are valid.

\section{Proof of "addition" theorems for $q$-linear grids}

In the case of $q$-linear grids, we can give a direct proof of (5.3) using the following version of the $q$-binomial theorem.

Lemma 6.1. If $x=x(z)=C_{1} q^{-z}$ and $y=x(s)=C_{1} q^{-s}$, then

$$
\left[x(z)-x\left(s+\frac{n-1}{2}\right)\right]^{(n)}=\sum_{k=0}^{n} \frac{\gamma(n) !}{\gamma(k) ! \gamma(n-k) !} x^{k}(-y)^{n-k} \text {. }
$$


Proof. For the $q$-linear lattice $x=C_{1} q^{-z}$, from the definition of the generalized power (2.5), one gets

$$
\left[x(z)-x\left(s+\frac{n-1}{2}\right)\right]^{(n)}=\sum_{k=0}^{n} c_{k} x^{k}(z)
$$

where $c_{k}$ are some constants independent of $x$. Let us apply the operator $\delta / \delta x(z)$ to both sides of (6.2) $m$ times. With the help of (2.6),

$$
\begin{aligned}
& \frac{\gamma(n) !}{\gamma(n-m) !}\left[x(z)-x\left(s+\frac{n-m-1}{2}\right)\right]^{(n-m)} \\
& =\sum_{k=m}^{n} c_{k} \gamma(k) \gamma(k-1) \cdots \gamma(k-m+1) x^{k-m}(z) .
\end{aligned}
$$

Substituting $x(z)=0$ (or $z \rightarrow-\infty$ when $|q|<1$ ),

$$
\frac{\gamma(n) !}{\gamma(n-m) !}\left[0-x\left(s+\frac{n-m-1}{2}\right)\right]^{(n-m)}=\gamma(m) ! c_{m}
$$

But from (2.5),

$$
\begin{aligned}
{[0-} & \left.x\left(s+\frac{n-m-1}{2}\right)\right]^{(n-m)} \\
& =(-1)^{n-m} \prod_{k=0}^{n-m-1} x\left(s+\frac{n-m-1}{2}-k\right) \\
& =(-1)^{n-m} x\left(s+\frac{n-m-1}{2}\right) \cdots x\left(s-\frac{n-m-1}{2}\right) \\
& =(-1)^{n-m} y^{n-m},
\end{aligned}
$$

which gives

$$
c_{m}=\frac{\gamma(n) !}{\gamma(m) ! \gamma(n-m) !}(-y)^{n-m}
$$

Combining (6.2) and (6.5), one gets (6.1).

We gave the proof of (6.1) in terms of generalized powers. Using (2.4) and (2.11), one can reduce (6.1) to a terminating form of the $q$-binomial theorem [9].

Theorem 6.2. Let

$$
u(x, y):=\sum_{n=0}^{\infty} \frac{\xi^{n}}{\gamma(n) !}\left[x(z)-x\left(s+\frac{n-1}{2}\right)\right]^{(n)}
$$

and

$$
u(x):=u(x, 0)=\sum_{n=0}^{\infty} \frac{\xi^{n}}{\gamma(n) !} x^{n}
$$

where $x=C_{1} q^{-z}$ and $y=C_{1} q^{-s}$. Then

$$
u(x, y)=u(x) u(-y)
$$


Proof. By (6.1) and (6.6)-(6.7),

$$
\begin{aligned}
u(x, y) & =\sum_{n=0}^{\infty} \frac{\xi^{n}}{\gamma(n) !}\left[x(z)-x\left(s+\frac{n-1}{2}\right)\right]^{(n)} \\
& =\sum_{n=0}^{\infty} \xi^{n} \sum_{k=0}^{n} \frac{x^{k}(-y)^{n-k}}{\gamma(k) ! \gamma(n-k) !} \\
& =\sum_{k=0}^{\infty} \xi^{k} \frac{x^{k}}{\gamma(k) !} \sum_{m=0}^{\infty} \xi^{m} \frac{(-y)^{m}}{\gamma(m) !} \\
& =u(x) u(-y)
\end{aligned}
$$

provided that all series converge.

Relation (5.3) is equivalent to (6.8) when $y \rightarrow-y$.

\section{General case: product formula}

In this section, we shall give an alternative proof of the "addition" theorem (3.9) on the basis of a more general product formula for the functions (2.3).

Lemma 7.1. The following symmetry relation holds

$$
\begin{aligned}
\sum_{k=0}^{n} & \frac{\left[x(z)-x\left(s+\frac{k-1}{2}\right)\right]^{(k)}}{\gamma(k) !} \frac{\left[x(r)-x\left(t+\frac{n-k-1}{2}\right)\right]^{(n-k)}}{\gamma(n-k) !} \\
& =\sum_{k=0}^{n} \frac{\left[x(z)-x\left(t+\frac{k-1}{2}\right)\right]^{(k)}}{\gamma(k) !} \frac{\left[x(r)-x\left(s+\frac{n-k-1}{2}\right)\right]^{(n-k)}}{\gamma(n-k) !}
\end{aligned}
$$

on the general q-quadratic lattice (2.2).

Proof. Let us mention first of all that (7.1) is true in two symmetric cases, when $t=s$ and $z=r$. If $n=1$, one gets

$$
[x(r)-x(t)]+[x(z)-x(s)]=[x(r)-x(s)]+[x(z)-x(t)],
$$

which also is correct.

Now we can prove (7.1) by induction. Denote the left-hand side of (7.1) as

$$
\begin{aligned}
L_{n}(z) & :=L_{n}(z, r, s, t) \\
& =\sum_{k=0}^{n} \frac{\left[x(z)-x\left(s+\frac{k-1}{2}\right)\right]^{(k)}}{\gamma(k) !} \frac{\left[x(r)-x\left(t+\frac{n-k-1}{2}\right)\right]^{(n-k)}}{\gamma(n-k) !} .
\end{aligned}
$$

By (2.6),

$$
\frac{\delta L_{n}(z)}{\delta x(z)}=L_{n-1}(z) .
$$

Denote also the difference between the left-and the right-hand sides of (7.1) as

$$
D_{n}(z):=L_{n}(z, r, s, t)-L_{n}(z, r, t, s)
$$


and suppose that $D_{n}(z)=0$, which means that (7.1) holds for some positive integer number $n$ by our assumption. Then, by (7.3),

$$
\frac{\delta D_{n+1}(z)}{\delta x(z)}=D_{n}(z)=0
$$

or,

$$
D_{n+1}(z)=C=\text { constant }
$$

because $D_{n+1}(z)$ is a polynomial in $x$ by definition. To find this constant, choose $z=r$ when $C=D_{n+1}(r)=0$. Thus, $D_{n+1}=0$, which proves our lemma by induction.

We can derive now the following product formula.

Theorem 7.2. Functions $u(z, s)$ defined by (2.3) satisfy

$$
u(z, s) u(r, t)=u(z, t) u(r, s)
$$

provided that all series converge.

Proof. By (2.3) and (7.1),

$$
\begin{aligned}
u(z, s) & u(r, t) \\
= & \sum_{k=0}^{\infty} \xi^{k} \frac{\left[x(z)-x\left(s+\frac{k-1}{2}\right)\right]^{(k)}}{\gamma(k) !} \sum_{m=0}^{\infty} \xi^{m} \frac{\left[x(r)-x\left(t+\frac{m-1}{2}\right)\right]^{(m)}}{\gamma(m) !} \\
= & \sum_{n=0}^{\infty} \xi^{n} \sum_{k=0}^{n} \frac{\left[x(z)-x\left(s+\frac{k-1}{2}\right)\right]^{(k)}}{\gamma(k) !} \frac{\left[x(r)-x\left(t+\frac{n-k-1}{2}\right)\right]^{(n-k)}}{\gamma(n-k) !} \\
= & \sum_{n=0}^{\infty} \xi^{n} \sum_{k=0}^{n} \frac{\left[x(z)-x\left(t+\frac{k-1}{2}\right)\right]^{(k)}}{\gamma(k) !} \frac{\left[x(r)-x\left(s+\frac{n-k-1}{2}\right)\right]^{(n-k)}}{\gamma(n-k) !} \\
= & \sum_{k=0}^{\infty} \xi^{k} \frac{\left[x(z)-x\left(t+\frac{k-1}{2}\right)\right]^{(k)}}{\gamma(k) !} \sum_{m=0}^{\infty} \xi^{m} \frac{\left[x(r)-x\left(s+\frac{m-1}{2}\right)\right]^{(m)}}{\gamma(m) !} \\
= & u(z, t) u(r, s)
\end{aligned}
$$

where $\xi=(-\lambda / \sigma)^{1 / 2}$.

Corollary 7.3. The "addition" theorem (3.9) is a special case of the product formula (7.7).

Proof. To get (3.9) from (7.7), choose $r=t=z_{0}$ where $q^{-z_{0}}=i \sqrt{\mu}$ and $\mu=C_{2} / C_{1}$ :

$$
u(z, s) u\left(z_{0}, z_{0}\right)=u\left(z, z_{0}\right) u\left(z_{0}, s\right) .
$$


But from (2.17),

$$
\begin{aligned}
& u\left(z, z_{0}\right)={ }_{2} \varphi_{1}\left(\begin{array}{c}
-\mu q^{1+2 z},-q^{1-2 z} / \mu \\
q
\end{array} q^{2},-\frac{\lambda C_{1} C_{2}(1-q)^{2}}{\sigma q^{1 / 2}}\right) \\
& +\left(-\frac{\lambda}{\sigma}\right)^{1 / 2} x(z)_{2} \varphi_{1}\left(\begin{array}{c}
-\mu q^{2+2 z},-q^{2-2 z} / \mu \\
q^{3}
\end{array} q^{2},-\frac{\lambda C_{1} C_{2}(1-q)^{2}}{\sigma q^{1 / 2}}\right), \\
& u\left(z_{0}, s\right)={ }_{2} \varphi_{1}\left(\begin{array}{c}
-\mu q^{1+2 s},-q^{1-2 s} / \mu \\
q
\end{array} q^{2},-\frac{\lambda C_{1} C_{2}(1-q)^{2}}{\sigma q^{1 / 2}}\right) \\
& -\left(-\frac{\lambda}{\sigma}\right)^{1 / 2} x(s)_{2} \varphi_{1}\left(\begin{array}{c}
-\mu q^{2+2 s},-q^{2-2 s} / \mu \\
q^{3} ; q^{2},-\frac{\lambda C_{1} C_{2}(1-q)^{2}}{\sigma q^{1 / 2}}
\end{array}\right) \text {, }
\end{aligned}
$$

and

$$
\begin{aligned}
u\left(z_{0}, z_{0}\right) & ={ }_{1} \varphi_{0}\left({ }_{-}^{q} ; q^{2},-\frac{\lambda C_{1} C_{2}(1-q)^{2}}{\sigma q^{1 / 2}}\right) \\
& =\frac{\left(-C_{1} C_{2}(1-q)^{2} q^{1 / 2} \lambda / \sigma ; q^{2}\right)_{\infty}}{\left(-C_{1} C_{2}(1-q)^{2} q^{-1 / 2} \lambda / \sigma ; q^{2}\right)_{\infty}}
\end{aligned}
$$

We have used the $q$-binomial theorem when $\left|C_{1} C_{2}(1-q)^{2} q^{-1 / 2} \lambda / \sigma\right|<1$. Comparing (2.17), (3.1), (3.5), and (7.9)-(7.11), one gets (3.9) from (7.8).

On the other hand, it is easy to check that (7.7) follows from (7.8). Therefore, the product formula (7.7) and the "addition" theorem (7.8) are really equivalent.

Corollary 7.4. Relations (7.8)-(7.11) give us the analytic continuation of the functions (2.17).

Proof. Indeed, both sides of (7.8) are well-defined when $\left|C_{1} C_{2}(1-q)^{2} q^{-1 / 2} \lambda / \sigma\right|<1$. But the analytic continuation of the ${ }_{2} \varphi_{1} \mathrm{~s}$ on the right side into a larger domain is easy to determine (see [9]). For example,

$$
\begin{aligned}
& { }_{2} \varphi_{1}\left(\begin{array}{c}
-\mu q^{1+2 z},-q^{1-2 z} / \mu \\
q
\end{array} q^{2}, \zeta\right) \\
& \quad=\frac{\left(-\mu q^{1+2 z} \zeta,-q^{1-2 z} \zeta / \mu ; q^{2}\right)_{\infty}}{\left(q, \zeta ; q^{2}\right)_{\infty}}{ }_{2} \varphi_{2}\left(\begin{array}{c}
\zeta, q \zeta \\
-\mu q^{1+2 z} \zeta,-q^{1-2 z} \zeta / \mu
\end{array} ; q^{2}, q\right)
\end{aligned}
$$


26

SUSLOV

$$
\begin{aligned}
& =\frac{\left(-q^{-2 z} / \mu,-q^{1-2 z} / \mu,-\mu q^{1+2 z} \zeta,-q^{1-2 z} / \mu \zeta ; q^{2}\right)_{\infty}}{\left(q, q^{-4 z} / \mu^{2}, \zeta, q / \zeta ; q^{2}\right)_{\infty}}
\end{aligned}
$$

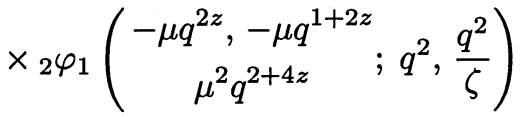

$$
\begin{aligned}
& +\frac{\left(-\mu q^{2 z},-\mu q^{1+2 z},-q^{1-2 z} \zeta / \mu,-\mu q^{1+2 z} / \zeta ; q^{2}\right)_{\infty}}{\left(q, \mu^{2} q^{4 z}, \zeta, q / \zeta ; q^{2}\right)_{\infty}} \\
& \times{ }_{2} \varphi_{1}\left(\begin{array}{c}
-q^{-2 z} / \mu,-q^{1-2 z} / \mu \\
q^{2-4 z} / \mu^{2}
\end{array} q^{2}, \frac{q^{2}}{\zeta}\right)
\end{aligned}
$$

and

$$
\begin{aligned}
& { }_{2} \varphi_{1}\left(\begin{array}{c}
-\mu q^{2+2 z},-q^{2-2 z} / \mu \\
q^{3} ; q^{2}, \zeta
\end{array}\right) \\
& =\frac{\left(-\mu q^{2+2 z} \zeta,-q^{2-2 z} \zeta / \mu ; q^{2}\right)_{\infty}}{\left(q^{3}, \zeta ; q^{2}\right)_{\infty}}{ }_{2} \varphi_{2}\left(\begin{array}{c}
\zeta, q \zeta \\
-\mu q^{2+2 z} \zeta,-q^{2-2 z} \zeta / \mu
\end{array} ; q^{2}, q^{3}\right) \\
& =\frac{\left(-q^{1-2 z} / \mu,-q^{2-2 z} / \mu,-\mu q^{2+2 z} \zeta,-q^{-2 z} / \mu \zeta ; q^{2}\right)_{\infty}}{\left(q^{3}, q^{-4 z} / \mu^{2}, \zeta, q / \zeta ; q^{2}\right)_{\infty}} \\
& \times{ }_{2} \varphi_{1}\left(\begin{array}{c}
-\mu q^{1+2 z},-\mu q^{2 z} \\
\mu^{2} q^{2+4 z}
\end{array} ; q^{2}, \frac{q^{2}}{\zeta}\right) \\
& +\frac{\left(-\mu q^{1+2 z},-\mu q^{2+2 z},-q^{2-2 z} \zeta / \mu,-\mu q^{2 z} / \zeta ; q^{2}\right)_{\infty}}{\left(q^{3}, \mu^{2} q^{4 z}, \zeta, q / \zeta ; q^{2}\right)_{\infty}} \\
& \times{ }_{2} \varphi_{1}\left(\begin{array}{c}
-q^{1-2 z} / \mu,-q^{-2 z} / \mu \\
q^{2-4 z} / \mu^{2}
\end{array} q^{2}, \frac{q^{2}}{\zeta}\right)
\end{aligned}
$$

where $\zeta=C_{1} C_{2}(1-q)^{2} q^{-1 / 2} \lambda / \sigma$.

Equations (7.8), (7.13), and (7.15) also determine the asymptotic behavior of the functions (2.17) for large values of their arguments.

Remark 7.1. According to (2.4),

$$
\gamma(n) !=q^{-n(n-1) / 4} \frac{(q ; q)_{n}}{(1-q)^{n}}
$$

and

$$
\frac{\gamma(n) !}{\gamma(k) ! \gamma(n-k) !}=q^{-(n-k) k / 2} \frac{(q ; q)_{n}}{(q ; q)_{k}(q ; q)_{n-k}}=(-1)^{k} q^{(n+1) k / 2} \frac{\left(q^{-n} ; q\right)_{k}}{(q ; q)_{k}} .
$$


Therefore, for the general $q$-quadratic lattice (2.2), relation (7.1) can be rewritten as the following sum of $q$-shifted factorials:

$$
\begin{aligned}
q^{-n t} \sum_{k=0}^{n} & (-1)^{k} q^{(t-s+(n+1) / 2) k} \frac{\left(q^{-n} ; q\right)_{k}}{(q ; q)_{k}} \\
& \times\left(q^{s-z-(k-1) / 2}, \mu q^{s+z-(k-1) / 2} ; q\right)_{k} \\
& \times\left(q^{t-r-(n-k-1) / 2}, \mu q^{t+r-(n-k-1) / 2} ; q\right)_{n-k} \\
=q^{-n s} & \sum_{k=0}^{n}(-1)^{k} q^{(s-t+(n+1) / 2) k} \frac{\left(q^{-n} ; q\right)_{k}}{(q ; q)_{k}} \\
& \times\left(q^{t-z-(k-1) / 2}, \mu q^{t+z-(k-1) / 2} ; q\right)_{k} \\
& \times\left(q^{s-r-(n-k-1) / 2}, \mu q^{s+r-(n-k-1) / 2} ; q\right)_{n-k}
\end{aligned}
$$

Here $\mu=C_{2} / C_{1}$.

\section{Linear grid: addition theorem}

In the case of the linear lattice, $x(z)=z$, the function (2.3) has the simple form

$$
u(z, s)=u(z-s)
$$

where

$$
\begin{aligned}
u(z)= & \sum_{n=0}^{\infty} \frac{\alpha^{n}}{n !}\left(z-\frac{n-1}{2}\right)_{n} \\
= & { }_{2} F_{1}\left(\begin{array}{c}
1 / 2+z, 1 / 2-z \\
1 / 2
\end{array} ;-\frac{\alpha^{2}}{4}\right) \\
& +\alpha z{ }_{2} F_{1}\left(\begin{array}{c}
1+z, 1-z \\
3 / 2
\end{array} ;-\frac{\alpha^{2}}{4}\right) .
\end{aligned}
$$

Here $(a)_{n}=a(a+1) \cdots(a+n-1)=\Gamma(a+n) / \Gamma(a)$ and ${ }_{2} F_{1}$ is the hypergeometric function.

The general product formula (7.7) takes the form

$$
u(z-s) u(r-t)=u(z-t) u(r-s)
$$

Also,

$$
u(0)=\sum_{k=0}^{\infty} \frac{(1 / 2)_{k}}{k !}\left(-\frac{\alpha^{2}}{4}\right)^{k}=\left(1+\frac{\alpha^{2}}{4}\right)^{-1 / 2}
$$


Therefore, for the function

$$
\begin{aligned}
e(z):=u(z) / u(0) & \\
=\left(1+\frac{\alpha^{2}}{4}\right)^{1 / 2} & {\left[{ }_{2} F_{1}\left(\begin{array}{c}
1 / 2+z, 1 / 2-z \\
1 / 2
\end{array}-\frac{\alpha^{2}}{4}\right)\right.} \\
& \left.+\alpha z_{2} F_{1}\left(\begin{array}{c}
1+z, 1-z \\
3 / 2
\end{array}-\frac{\alpha^{2}}{4}\right)\right],
\end{aligned}
$$

the product formula (8.3) has the form of the usual addition theorem,

$$
e(z+s)=e(z) e(s) \text {. }
$$

But the only measurable solution of this functional equation is $\exp (\beta z)$, so

$$
e(z)=e^{\beta z} \text {. }
$$

To find the value of the constant $\beta$, one can choose $z=1 / 2$ which gives

$$
\beta=2 \log \left(\left(1+\frac{\alpha^{2}}{4}\right)^{1 / 2}+\frac{\alpha}{2}\right) .
$$

Thus,

$$
e(z):=e(z ; \alpha)=\left(\left(1+\frac{\alpha^{2}}{4}\right)^{1 / 2}+\frac{\alpha}{2}\right)^{z}
$$

The direct transformation of (8.5) to (8.9) can be done with the help of the relations (15.1.16) and (15.1.18) of [1]. Therefore, the "difference" exponential function (8.5) coincides with the classical exponential function.

Changing $\alpha=i \omega$ in (8.5) and (8.9), we get

$$
\cos \left(z \arctan \left(\frac{\omega / 2}{\left(1-\omega^{2} / 4\right)^{1 / 2}}\right)\right)=\left(1-\frac{\omega^{2}}{4}\right)^{1 / 2}{ }_{2} F_{1}\left(\begin{array}{c}
1 / 2+z, 1 / 2-z \\
1 / 2
\end{array} ; \frac{\omega^{2}}{4}\right)
$$

and

$$
\sin \left(z \arctan \left(\frac{\omega / 2}{\left(1-\omega^{2} / 4\right)^{1 / 2}}\right)\right)=\left(1-\frac{\omega^{2}}{4}\right)^{1 / 2} \omega z_{2} F_{1}\left(\begin{array}{c}
1+z, 1-z \\
3 / 2
\end{array} ; \frac{\omega^{2}}{4}\right)
$$

as "difference" analogs of $\cos \omega z$ and $\sin \omega z$. Addition formulas (1.15)-(1.16) hold.

\section{Application: continuous $q$-Hermite polynomials}

Let us introduce the following polynomials $H_{n}(x, y \mid q)$ in two variables,

$$
\begin{aligned}
& H_{2 n}(x, y \mid q)=(-1)^{n} \frac{\left(q ; q^{2}\right)_{n}}{q^{n}} \\
& \quad \times{ }_{6} \varphi_{5}\left(\begin{array}{c}
q^{-n},-q^{-n},-q^{1 / 2} e^{i \theta+i \varphi},-q^{1 / 2} e^{i \theta-i \varphi},-q^{1 / 2} e^{i \varphi-i \theta},-q^{1 / 2} e^{-i \theta-i \varphi} \\
-q, q^{1 / 2},-q^{1 / 2}, 0,0
\end{array} ; q, q^{2}\right)
\end{aligned}
$$


and

$$
\begin{aligned}
& H_{2 n+1}(x, y \mid q)=(-1)^{n} 2 \frac{\left(q^{3} ; q^{2}\right)_{n}}{q^{2 n}}(\cos \theta+\cos \varphi) \\
& \quad \times{ }_{6} \varphi_{5}\left(\begin{array}{c}
q^{-n},-q^{-n},-q e^{i \theta+i \varphi},-q e^{i \theta-i \varphi},-q e^{i \varphi-i \theta},-q e^{-i \theta-i \varphi} \\
-q, q^{3 / 2},-q^{3 / 2}, 0,0
\end{array} ; q, q^{2}\right)
\end{aligned}
$$

with $x=\cos \theta$ and $y=\cos \varphi$, as an extension of the continuous $q$-Hermite polynomials $H_{n}(x \mid q)$ (see, for example, $\left.[5,9]\right)$. One can see that

$$
H_{n}(x, 0 \mid q)=H_{n}(x \mid q)
$$

and we may interpret $H_{n}(x, y \mid q)$ as a $q$-version of the Hermite polynomials $H_{n}(x+y)$ with the shifted argument.

It is easy to see that the $q$-exponential function (3.1) is a generating function for the polynomials $H_{n}(x, y \mid q)$.

Theorem 9.1. We have

$$
\begin{aligned}
& \sum_{n=0}^{\infty} \frac{q^{n^{2} / 4}}{(q ; q)_{n}} \alpha^{n} H_{n}(x, y \mid q)=\left(q \alpha^{2} ; q^{2}\right)_{\infty} \mathcal{E}_{q}(x, y ; \alpha) \\
& \quad=\left(\alpha^{2} ; q^{2}\right)_{\infty} \sum_{n=0}^{\infty} \frac{q^{n^{2} / 4}}{(q ; q)_{n}} e^{-i n \varphi}\left(-q^{(1-n) / 2} e^{i \varphi+i \theta},-q^{(1-n) / 2} e^{i \varphi-i \theta} ; q\right)_{n} \alpha^{n}
\end{aligned}
$$

Proof. Substitute (9.1) and (9.2) into the left side of (9.4) and interchange the order of summation with the help of [9, I.12 and II.2]. As a result, we get the right side of (9.4) due to (3.1).

Corollary 9.2. The continuous $q$-Hermite polynomials have the generating relation

$$
\sum_{n=0}^{\infty} \frac{q^{n^{2} / 4}}{(q ; q)_{n}} \alpha^{n} H_{n}(x \mid q)=\left(q \alpha^{2} ; q^{2}\right)_{\infty} \mathcal{E}_{q}(x ; \alpha) .
$$

Proof. Let $y=0$ in (9.4).

See also [10]. Relation (9.5) is a $q$-version of the generating function

$$
\sum_{n=0}^{\infty} \frac{\alpha^{n}}{n !} H_{n}(x)=e^{2 \alpha x-\alpha^{2}}
$$

for the Hermite polynomials [17, 19]. The limit $q \rightarrow 1^{-}$is obvious if we rewrite the right side of $(9.5)$ as $\mathcal{E}_{q}(x, \alpha) E_{q^{2}}\left(-q \alpha^{2}\right)$ where $E_{q}(z)$ is one of Jackson's $q$-exponential functions defined by $[9$, II.2].

\section{Corollary 9.3.}

$$
\begin{aligned}
H_{n}(x, y \mid q)= & \sum_{k=0}^{[n / 2]}(-1)^{k} \frac{(q ; q)_{n}}{\left(q^{2} ; q^{2}\right)_{k}(q ; q)_{n-2 k}} q^{k(2 k-n-1)} \\
& \times e^{-i(n-2 k) \varphi}\left(-q^{(1-n+2 k) / 2} e^{i \varphi+i \theta},-q^{(1-n+2 k) / 2} e^{i \varphi-i \theta} ; q\right)_{n-2 k}
\end{aligned}
$$


Proof. By [9, II.2],

$$
\left(\alpha^{2} ; q^{2}\right)_{\infty}=\sum_{k=0}^{\infty} \frac{q^{k(k-1)}\left(-\alpha^{2}\right)^{k}}{\left(q^{2} ; q^{2}\right)_{k}} .
$$

Using (9.8) in the right side of (9.4) and equating coefficients of $\alpha^{n}$ on both sides, one gets (9.7).

Relation (9.7) is clearly an extension of

$$
H_{n}(x+y)=\sum_{k=0}^{[n / 2]} \frac{(-1)^{k} n !}{k !(n-k) !}(2 x+2 y)^{n-2 k},
$$

see $[17,19]$. Also, the following extension of equation (4) from section 110 of [17] holds.

\section{Corollary 9.4.}

$$
\begin{aligned}
& \left(-q^{(1-n) / 2} e^{i \varphi+i \theta},-q^{(1-n) / 2} e^{i \varphi-i \theta} ; q\right)_{n} e^{-i n \varphi} \\
& =\sum_{k=0}^{[n / 2]} q^{k(k-n)} \frac{(q ; q)_{n}}{\left(q^{2} ; q^{2}\right)_{k}(q ; q)_{n-2 k}} H_{n-2 k}(x, y \mid q) .
\end{aligned}
$$

Proof. Multiply both sides of (9.4) by

$$
\sum_{k=0}^{\infty} \frac{\alpha^{2 k}}{\left(q^{2} ; q^{2}\right)_{k}}=\frac{1}{\left(\alpha^{2} ; q^{2}\right)_{\infty}}
$$

(see $\left[9\right.$, II.1]) and equate coefficients of $\alpha^{n}$.

Corollary 9.5. Polynomials $H_{n}(x, y \mid q)$ have the difference-differentiation formulas

$$
\begin{aligned}
\frac{\delta H_{n}(x, y \mid q)}{\delta x} & =\frac{\delta H_{n}(x, y \mid q)}{\delta y} \\
& =2 q^{(1-n) / 2} \frac{1-q^{n}}{1-q} H_{n-1}(x, y \mid q) .
\end{aligned}
$$

Proof. Apply operators $\delta / \delta x$ and $\delta / \delta y$ to the both sides of (9.4) using (3.8) and compare coefficients of $\alpha^{n}$.

The "addition" formula (3.9) gives us the ability to express the polynomials $H_{n}(x, y \mid q)$ in terms of the continuous $q$-Hermite polynomials $H_{n}(x \mid q)$.

\section{Theorem 9.6.}

$$
\begin{aligned}
H_{n}(x, y \mid q)=\sum_{m, k} & \frac{(q ; q)_{n}}{\left(q^{2} ; q^{2}\right)_{k}(q ; q)_{m}(q ; q)_{n-m-2 k}} q^{k(m-n+k+1)+m(m-n) / 2} \\
& \times H_{m}(x \mid q) H_{n-m-2 k}(y \mid q) .
\end{aligned}
$$


Proof. Due to (9.4)-(9.5) and (3.9),

$$
\begin{aligned}
\sum_{n=0}^{\infty} \frac{q^{n^{2} / 4}}{(q ; q)_{n}} \alpha^{n} H_{n}(x, y \mid q)= & \left(q \alpha^{2} ; q^{2}\right)_{\infty} \mathcal{E}_{q}(x ; \alpha) \mathcal{E}_{q}(y ; \alpha) \\
= & \sum_{k=0}^{\infty} \frac{\alpha^{2 k} q^{k}}{\left(q^{2} ; q^{2}\right)_{k}} \sum_{m=0}^{\infty} \frac{q^{m^{2} / 4}}{(q ; q)_{m}} \alpha^{m} H_{m}(x \mid q) \\
& \times \sum_{p=0}^{\infty} \frac{q^{p^{2} / 4}}{(q ; q)_{p}} \alpha^{p} H_{p}(y \mid q) .
\end{aligned}
$$

We have used (9.10) with $\alpha \rightarrow \alpha q^{1 / 2}$. By equating coefficients of $\alpha^{n}$ on both sides, we obtain (9.12).

Using the decomposition (9.12) for $H_{n}(x, y \mid q)$, one can give a direct proof of (9.11) by applying the difference-differentiation formula for the continuous $q$-Hermite polynomials $H_{n}(x \mid q)$. Polynomials $H_{n}(x, y \mid q)$ deserve more detailed consideration.

Acknowledgments. I would like to thank Dick Askey for encouraging conversations and also for presenting my talk on a related topic at the Montréal meeting on "Algebraic Methods and $q$-Special Functions", May 1996. I wish to thank Joaquin Bustoz, George Gasper, Mourad Ismail, John McDonald, and Mizan Rahman for valuable discussions and comments. I would like to thank T. Koornwinder and H. T. Koelink for pointing out the references [7, 13]. This paper was prepared while the author visited the Department of Mathematics at Arizona State University, and I gratefully acknowledge their hospitality.

\section{References}

1. M. Abramowitz and I. A. Stegun (eds), Handbook of Mathematical Functions, Dover, New York, 1972.

2. R. A. Askey, M. Rahman, and S. K. Suslov, On a general q-Fourier transformation with nonsymmetric kernels, J. Comp. Appl. Math. 68 (1996), 25-55.

3. R. Askey and S. K. Suslov, The q-harmonic oscillator and an analogue of the Charlier polynomials, J. Phys. A26 (1993), L693-L698.

4. 29 (1993), 123-132.

5. R. A. Askey and J. A. Wilson, Some basic hypergeometric orthogonal polynomials that generalize Jacobi polynomials, Memoirs Amer. Math. Soc. 319 (1985).

6. N. M. Atakishiyev and S. K. Suslov, Difference hypergeometric functions, In: Progress in Approximation Theory: An International Perspective (Eds. A. A. Gonchar and E. B. Saff), Springer Series in Computational Mathematics, Vol. 19, Springer-Verlag, 1992, pp. 1-35.

7. H. Exton, q-Hypergeometric Functions, Ellis Horwood, 1983.

8. R. Floreanini and L. Vinet, On the quantum group and quantum algebra approach to q-special functions, Lett. Math. Phys. 27 (1993), 179-190.

9. G. Gasper and M. Rahman, Basic Hypergeometric Series, Cambridge University Press, Cambridge, 1990.

10. M. E. H. Ismail and R. Zhang, Diagonalization of certain integral operators, Advances in Math. 108 (1994), 1-33.

11. M. E. H. Ismail, M. Rahman, and R. Zhang, Diagonalization of certain integral operators II, J. Comp. Appl. Math. 68 (1996), 163-196.

12. E. G. Kalnins and W. Miller, Models of $q$-algebra representations: $q$-integral transforms and "addition" theorems, J. Math. Phys. 35 (1994), 1951-1975. 
13. T. H. Koornwinder and R. F. Swarttow, On q-analogs of the Fourier and Hankel transform, Trans. Amer. Math. Soc. 333 (1992), 445-461.

14. A. F. Nikiforov, S. K. Suslov, and V. B. Uvarov, Classical Orthogonal Polynomials of a Discrete Variable, Nauka, Moscow, 1985 (in Russian); English translation, Springer-Verlag, Berlin, 1991.

15. M. Rahman, The q-exponential functions, old and new, to appear.

16. M. Rahman and S. K. Suslov, Singular analogue of the Fourier transformation for the AskeyWilson polynomials, In: Symmetries and Integrability of Difference Equations, D. Levi et. al., eds., CRM Proceedings and Lecture Notes 9, Amer. Math. Soc., 1996, pp. 289-302.

17. E. D. Rainville, Special Functions, Chelsea, Bronx, 1971.

18. S. K. Suslov, The theory of difference analogues of special functions of hypergeometric type, Russian Math. Surveys 44 (1989), 227-278.

19. G. Szegö, Orthogonal Polynomials, Fourth Edition, Amer. Math. Soc., Providence, 1975.

KuRchatov Institute, Moscow 123182, Russia

Current address: Department of Mathematics, Arizona State University, Tempe, Arizona 852871804, USA

E-mail: suslov@math.la.asu.edu 